(C)2008 IEEE. Personal use of this material is permitted. However, permission to reprint/republish this material for advertising or promotional purposes or for creating new collective works for resale or redistribution to servers or lists, or to reuse any copyrighted component of this work in other works must be obtained from the IEEE. 


\title{
Highlighting the Issues in making an Informed Interaction-based Decision in Industrial Ecosystems
}

\author{
Omar Hussain, Tharam Dillon, Elizabeth Chang and Farookh Hussain \\ Digital Business Ecosystems and Business Intelligence Institute \\ Curtin University of Technology \\ Omar.Hussain@postgrad.curtin.edu.au
}

\begin{abstract}
In the literature, the notion of trust has been widely discussed and various approaches have been proposed to assess and quantify it, in order to take it into consideration when making an interaction-based decision in industrial ecosystems. At the same time, the notion and importance of risk have been widely ignored and not been acknowledged as an important factor to consider, along with trust; while making an interaction-based decision. Although there are approaches in the literature which attempt to quantify risk in the domain of a business interaction in industrial ecosystems, none of them proposes a methodology by which the initiating agent of the interaction can ascertain and determine all the various concepts that are expressed by risk in that domain, and then utilize it to make an informed interaction-based decision. In this paper we present an evaluation of the approaches from the literature and highlight the issues which need to be addressed in order to assist the initiating agent of the interaction in making an informed interaction-based decision with another agent in Industrial ecosystems.
\end{abstract}

\section{INTRODUCTION}

The rapid pace of development of the Internet has provided its users in the domain of business ecosystems with sophisticated technologies which ease the process of carrying out their activities. The term activities in this domain refers to providing the functionalities which enables the users to collaborate and interact with each other seamlessly. This enables businesses to complete their tasks in less time and reduce the delays associated with the conventional method of interactions, while at the same time boosting their efficiency and helping them to improve their sales, productivity and economy. But as mentioned by Chang et al. [1] 'The dynamic, open and convenient web environment not only boosts business potential and the economy but also creates concerns of security, trust, privacy and risks'. So the users, before utilizing the provided facilities to their advantage, should consider and analyze these aspects in order to make sure that they achieve what they desire, or get the maximum output in their interactions. Added to the fact, industrial ecosystems is mainly about the people or companies that populate a given interaction infrastructure. And when an interaction is being carried out in such an environment, it is up to the interacting entity to make sure that it proceeds in an interaction in which it hopes to achieve maximum benefit and interaction experience. But some important considerations for the users in such interactions are the notions of 'security', 'trust' and 'perceived risk' - in other words, those factors which help to make the interactions over this paradigm safe, secure and informed. This is because an agent in such environments has to make its' own decisions and has to be responsive and proactive when doing so. In this paper we do a survey of the literature and highlight the areas that need to be addressed in order for the initiating agent of the interaction in business ecosystems to make an informed interaction-based decision. Further in this paper whenever we use the terrn 'interaction' we refer it to a business interaction carried out in the domain of industrial ecosystems. This paper is organized into $\mathrm{V}$ sections. In section II we discuss the inter-relationship between security, trust and risk while decision-making in business interactions. In Section III we highlight the importance of perceived risk in considering it as an important concept while making an interaction-based decision. In Section IV we do an evaluation of approaches from the literature and then identify the issues that need to be addressed in order for the making an informed decisionmaking in industrial ecosystems. Finally in Section $\mathrm{V}$ we conclude the paper.

\section{Relationship BetweEn SECURITy, Trust AND Risk}

Security is a crucial aspect of any interaction. Security is the act of taking appropriate measures by which the threats or the dangers in the interaction are addressed, checked or eliminated. In other words, security means securing or protecting the users of the transaction from anything which might present a threat to the individuals involved in it. In today's advanced developing world, Internet has replaced the traditional paper-based way of exchanging information of the past, thereby enabling the information either by the user or the business to be sent in a much more efficient, fast and reliable way. The information which the businesses own is a very important resource for them as it forms the essence of their development. As the interactions are being conducted online on the Internet, besides keeping the information that they have secure, businesses also have to consider providing security for the information that they acquire while interacting with their customers over the internet. In the context of this paper, security relates to the process of providing a secure environment or a secure space in the open architecture of the web, where the customers exchange information with other entities or businesses with an assurance that the information which they share and utilize is being sent only to, and by, the intended people, and that the information is not being changed in any way. To achieve this, businesses have to incorporate and provide their users with the latest technologies available, thereby improving the security they provide to the customers and enhancing their 
reputation. Enhancing the security helps the businesses to protect their users from intrusions, attacks from the outside world and safeguards them from vulnerabilities, subsequently increasing the confidence of the customers/users. The failure of a business to address these concerns might affect its chances of sustainability in the environment by jeopardizing return business or acquisition of new customers.

Hence security in a transaction is utilized to efficiently shield the environment so that users and businesses can interact without disruptive forces affecting the interaction. In other words, security is the first aspect considered by customers who take part in an online interaction. It acts as a barrier to the outside forces which aim to disrupt the interaction, and enables the user of the interaction to initiate the process of ascertaining whether or not to place his/her 'trust' in the other interacting agent. The word 'trust' here can be interpreted in different ways according to its context. The concept of 'Trusted Computing' has been proposed in the literature in an attempt to solve some of the security problems encountered in today's world by adopting the security mechanisms [2]. In this context, the word 'trust' is related to trusting a business based on the security mechanisms that it provides, and it refers to the perception of the human individual in 'trusting' or in other terms 'considering' the business as a possible entity with which s/he may decide later whether to interact with or not. This notion of trust is quite different from what is required in the context of a business interaction when an entity actually decides to interact with the other entity, and where we mean that 'an entity trusts another entity in the following interaction'. In this context, the word 'trust' refers to the level of confidence that an entity has in the other entity's ability to achieve its desired outcomes through the interaction. Although the word 'trust' is used in both instances, because the contexts are different, the word assumes quite a different meaning in each context. In the context of trusted computing, trust refers to the interacting entity accepting the provided security mechanisms adopted by the business in the interaction by which it feels safe and not vulnerable to the outside forces which might hamper its interaction. But this level of trust does not in any way mean that the entity will interact with that business or the other entity as it satisfies the level of trusted computing. The level of trust in trusted computing just helps the entity to overcome the initial barrier related to the security mechanisms in the business; in no way does it provide a guarantee or assurance that the business will act in a benevolent way and will behave such that the entity will receive the outcome(s) that it desires from the interaction. In order for that entity to have the level of trust necessary in order to really commit itself to an interaction with the business, within the business context, it needs to believe that the business can achieve its desired outcomes. In this context, the notion of trust has little bearing on the security provided by the business. In the context of business interactions in industrial ecosystem architecture, trust refers to analyzing the business' reputation and, based on that, determining its confidence or its level of belief in the capability of the business to provide or give what the user wants as a result of the interaction. Hence, a clear distinction can be drawn between trust and security in the context of decision making in industrial ecosystem business interactions. From the above discussion, it can be concluded that a customer/user takes the initial step of ascertaining the level of security provided by a business or entity before deciding whether or not to enter into an interaction, as security helps to provide a secure environment within which an interaction can be confidently carried out. Based on the level of security, the initiating entity can determine the level of belief and the level of risk in business interactions to make an informed decision about its interaction with the business or any other entity.

Risk highlights the threats and the level of loss in the interaction. As with the cross relationship between security and trust discussed earlier, the same holds for the relationship of risk with security and trust as discussed in the literature. In the context of a business interaction, analyzing risk in the area of security will only ascertain the security risks present in the interaction. Such risks will not provide any meaningful information to the entity or agent while making an informed interacting based decision in a business interaction, as this analysis would only highlight the areas in which the security risks of the business are present. In such scenarios, even if an entity considers the level of risks in the areas related to security, privacy as acceptable, then based on that level of risk, it cannot make an informed decision in interacting with the other entity in the context of a business interaction, as the level of risk determined in those areas is not synonymous with the level of risk to be determined for decision making in the context of business interactions in service oriented architecture. Similarly, another confusion present in the literature is in relation to the relationship between trust and risk; which considers trust to be the authoritative concept to risk, and that based only on the level of trust the decision making process in the business interaction can be carried out as it also represents or nullifies the level of risk in the interaction. But trust and risk represent different concepts depending on the different areas that are targeted. In generic terms, trust in the context of business interaction, shows the level of belief that an entity has in the other entity or business, but this level of belief does not express the degree and the magnitude of loss in the interaction. Such concepts can be determined only by the analysis of risk in the interaction in that context. We will explain the relationship between trust and risk further in the next section. In this paper, we do not aim to analyze the security aspects of the interaction which the initiating entity analyses in order to overcome the initial barrier of choosing those businesses or entities with which it may possibly interact in an industrial ecosystem domain. Moreover by doing a review of the literature, we conclude that various approaches have been proposed by which an initiating entity can analyze and assess the level of trust in the interaction and as a result in this paper we do not aim to determine the level of trust in the interaction which the initiating entity analyses to determine its level of belief in the other entity or business when it has to make an informed decision of choosing a business with which to interact. The scope of this paper covers the initiating entity of the interaction in order to analyze the level of risk 
semantically and qualitatively in a business interaction, by which it can more confidently make an informed interaction based decision with a business or entity; or choose which business or entity with which to interact from a given probable set. In the next section, we will study the importance of 'perceived risk' in an interaction, the relationships between trust and risk, and the effect that these concepts have on the behavior of an entity while making a decision in the business interaction context.

\section{Highlighting the Importance Of Perceived Risk}

In this section, we will discuss the importance of 'Perceived Risk' in an interaction. As mentioned in the last section, due to the ever-developing technologies in facilitating business interactions, users are increasingly becoming more attracted to and dependent on taking advantage of the facilities that are provided for them, and minimizing their efforts in doing the same thing by the old fashioned and hard way. Due to the ease of use and dependence of the users on the provided functionalities, some users become negligent and use the facilities in a rash way without weighing the benefits with the associated outcomes. And as it is common in today's world, there are some deceitful users who try to exploit others for their own benefit. The fact that such interactions are conducted in a widelydistributed, open and decentralized environment makes the tasks of such users much easier. Some users can foresee it and avoid such interactions, but some careless users pay the price for their negligence. Some users are skeptical and want to balance the advantages and disadvantages before the interaction, and hence need some concepts which can help them to achieve this. These concepts in the interaction can be determined by the initiating entity by ascertaining beforehand its level of belief, along with the possible loss that it would experience in achieving what it desires in the interaction, i.e. by assessing the level of 'trust' and 'risk' in the interaction. 'Trust' and 'risk' are significant concepts in today's world across all disciplines. Although significant, these terms are very old; and they have not been recently developed to combat such scenarios, but have been adapted from other fields. As pointed out by Koutrouli et al. [3] 'Trust in computer science is a concept that has been borrowed from the human society where people constantly apply it in their interactions'.

Although various conceptualizations of the term 'trust' have been presented in the literature, a generic definition of trust adopted from Chang et al. [1] is 'the belief or faith that a person or agent has in the another person or agent with respect to certain activities at a given time'. But as discussed earlier, this is not the only concept for an entity to consider while making an informed decision in an industrial ecosystem business interaction. Another important perception to consider in such interactions is the notion of 'perceived risk', i.e. identifying the level of failure and the possible losses in the transaction conducted in an industrial ecosystem interaction as there is a lack of a central authority between them. It has been well argued in the literature that perceived risk is an important factor in deterring a user from shopping online on the Internet [4-7]. To overcome this, researchers have adopted the notion of trust in the field of computer science. Based on the established level of trust, the researchers have tried to combat the perceived risk in the interaction. But in a business interaction, the terms trust and risk refer to two concepts, each of which must be considered individually when making a decision about an interaction. These two concepts help the initiating entity of the interaction to analyze, ponder and then decide its future course in the interaction with any other entity or business. In other words, these two concepts have an effect on the attitude or behavior of an entity while making a decision about whether or not to enter into an interaction with another entity.

Trust and risk are the two concepts associated with an interaction, which complement each other when making an informed decision about the future course of action. Both of these concepts, although complementing each other, express different meanings, which in turn are not interchangeable and cannot be substituted in achieving their respective outputs. Hence, it is incorrect to compare them and decide which one of them is the more important for better decision making in an interaction. In the literature, Mayer et al. [8] highlight the confusion in the relationship between risk and trust by stating "it is unclear whether risk is an antecedent to trust, is trust, or is an outcome of trust". It depends on how the entity or agent sees the relationship between trust and risk. Due to the importance of these terms, various relationships have been proposed between them in the literature. Depending on the relationships, the effect that they have on the behavior of the agent varies. As summarized by Gefen et al. [9], the relationship between Trust and Risk in an interaction can be classified into three main broad categories.

- Mediating relationship

- Moderating relationship

- Independent relationship.

But no matter whatever relationship is present between trust and perceived risk in the interaction, from the above discussion it can be concluded that both these concepts are important and play a significant role in an agent's decision about its behavior in the interaction and whether or not to take the interacting activity with any other agent or business. So for decision making in business interactions, it is very important for the interaction initiating entity to assess the perceived risk in interacting with another entity or business as, apart from just assessing trust, it determines the possible level of failure and degree of loss in the interaction. This is not given by the notion of trust whose generic interpretation refers only to the belief that a consumer has in the other entity in providing its desired outcomes.

As their conceptualization, the way trust and risk are quantified, and the output concepts that they give in an interaction for decision making also varies. Trust in an interaction can be expressed in probability in terms of belief, whereas perceived risk is best understood when expressed in terms of probabilities under conditions of uncertainty, which tend to express any possible loss in an interaction. As both these concepts are expressed in terms of probability, it may be possible that they both express the probability related to a 
certain event, but the types of outcomes that each concept expresses for that event are different from each other. While trust refers to the assessed belief or probability of having a desirable outcome performed or achievable in an event, risk refers to the assessed probability of not having a desirable outcome performed or achieved in that event, and the related outcomes when not attaining the desired outcome. In this sense, the terms 'trust' and 'risk' are opposite concepts. By expressing trust and risk as probabilities in an interaction, the magnitude of the effect that they have on the expressed outcomes can also be determined according to the probability of their occurrence. In other words, trust and risk are two concepts whose evaluation would give two contrasting perspectives of occurrence of an event, which in fact could be mirror images of each other. But in broad terms, the manner in which they are analyzed, and the outcomes of evaluating them, are not simply the converse of each other. Rather, they provide various insights which might help to resolve or allay the initiating agent's various doubts or suspicions which are present before the interaction, prior to making an informed decision about whether or not to interact with the other agent or business. Approaches have been proposed in the literature which relates perceived risk as a result of inadequate security and privacy in the interaction. Various approaches have been proposed which link perceived risk with the level of trust or the trustworthiness of the agent, which in turn is dependent on the security and privacy aspects of the interaction. But analyzing perceived risk in such areas will only highlight the shortcomings of these concepts, which leads to identifying their inherent risks such as security risk etc. But such risks do not express the actual concepts required as a result of risk analysis for decision making in business interactions. Further, some approaches in the literature propose to assess the perceived risk in business interaction for decision making, by utilizing the level of trust in it. But such approaches do not take into account and present the complete aspects which are to be expressed by the level of perceived risk in the domain of business interactions for taking an interacting decision. Hence, we propose that in order to study the effect of perceived risk on the behavior of the agent while making an interaction-based decision in business interactions, it should be assessed according to its object of analysis in that domain.

In the next section, we evaluate and highlight the approaches from the current literature which assist in decision-making in business interactions, and highlight the issues to be addressed in order for the interaction initiating agent to make an informed interaction-based decision.

\section{IDENTIFYING THE ISSUES TO BE ADDRESSED For MAKING AN INTERACTION-BASED DECISION IN INDUSTRIAL ECOSYSTEMS}

Our aim in this section is to build towards an integrative view in order to discover and identify the main issues in the literature that need to be addressed so as to enable an initiating agent of the interaction to make an informed decision of an interaction with another agent. We achieve this by evaluating the approaches from the literature which deals addresses the approaches for decision-making in an interaction. In the literature, various approaches have been proposed which assess the level of 'trust' for collaboration between agents in different areas related to the interaction. For example, trust has been analyzed in the security aspects of the interaction, in the scenario of taking an interactingbased decision, in the neurological aspects related to the interaction etc., by using various quantitative and qualitative methods and then has been utilized for decision making in the interaction. But no matter what approach is utilized for determining the level of trust in the interaction, none of them provides a complete methodology to model and represent all the aspects required for making an informed interacting-based decision. By the term 'complete methodology' we mean that, along with just considering the level of trust in the interaction, also taking into account the level and the magnitude of possible expected loss, which is expressed by the level of perceived risk and which forms a very important perspective while making an informed decision in the interaction. The level and magnitude of trust which those approaches assess and represent in an interaction will represent the utmost level of belief that an agent has in the other agent, or the degree of reliability to which an agent can depend on other agent, or the credibility of an agent to perform a given task in the interaction, which is ascertained, named and expressed according to its interpretation by the researchers in their approaches. But the degree of trust in the interaction omits and does not represent the 'degree or magnitude of loss' in the interaction. Also, from the evaluation of the approaches discussed in Appendix A which analyze the level of trust in the interaction, it can be inferred that the need for and importance of analyzing the concept of 'perceived risk' has been undermined and ignored. For example, under the trust establishment by using the security aspects category, Datta et al. [10] [11] consider security as the key to any interaction infrastructure, and trust as one of its constituent but they do not mention perceived risk as one of the key constituents for decision making. Aberer et al. [12] however, consider the possibility of agent ' $A$ ' cheating in the interaction, but they do not take into consideration the perceived risk while decision making, which gives the degree and magnitude of loss in interacting with agent ' $A$ ' due to its cheating. Damiani et al. [13, 14] propose an approach by which a peer chooses another peer with which to interact by the level of trust ascertained by polling. But they do not consider the notion of loss in their proposed approach XRep, when a peer decides in interacting with another peer. Similarly among the approaches of ascertaining trust in the interaction by using Bayesian approach, none of the approaches consider the importance of perceived risk and determine it in their approaches either while taking an interaction-based decision or in the other aspects related to their proposed approach. In the quantitative trust measurement approaches, although Hussain et al. [15] [1, 16] determine trust in the interaction according to its contextspecific and dynamic property, they do not consider the notion of perceived risk and possible loss when an agent decides to interact with another agent. Zheng et al. [17] considers the cost and utility function associated with an 
interaction while decision making, but they associate the cost with the 'rewards' of the consequences of decision. They do not consider the notion of loss associated with the uncertainty in the interaction and the consequences of decision. Although some researches highlight the importance and the need to analyze risk for better decision making along with just ascertaining the level of trust in the interaction, while proposing their approach they consider just the level of trust in the interaction for decision making [17, 18]. Among the other approaches which measure trust in the interaction for decision making, Akhter at al. [19] and Josang et al. [20] considers trust as the only factor in their approaches which motivates an agent in the interaction. Although Josang et al. [20] consider in their model the 'possible harm' and 'negative consequences' but they do not consider perceived risk in their model as a factor to address this and utilize it in decision making. Hassell [21] proposes a method whereby an agent decides to trust another agent by considering the neurological and socialization characteristics of its brain apart from just considering the 'level of belief' expressed by trust, but they fail to consider the importance of perceived risk and its impact on an agent's brain while decision making. Similarly, Pearson et al. [22] proposes an approach of making trustbased decision which omits taking perceived risk into consideration. Also, as discussed in the evaluation of the approaches, not all of them ascertain trust according to its multi-dimensional characteristics, and the perceived risk determined by utilizing this level of trust will not be according to its context-dependent, criteria-dependent and dynamic characteristics.

As discussed earlier in the paper, with the evolving and ever developing technology of the modern age internet for facilitating business interactions, the users would want to analyze the detail aspects of their interaction before taking an interacting-based decision. They would rather avoid deciding on an interaction from the one-dimensional perspective analysis of the interaction, i.e. just based on the level of belief or trust that an agent has in interacting with the other agent; rather, along with it they would also want to take into consideration each possible outcome and the associated costs to them, while making an informed interaction-based decision. This is done by analyzing the level of failure along with the costs associated with those failures in the interaction, apart from just considering the level of belief in the interaction. The importance of determining the level and degree of failure and the associated costs with them which constitutes the loss in the interaction, cannot be substituted or overcome by just determining the level of trust. Measuring the loss or impact in the interaction is within the scope of perceived risk in the interaction, which cannot be compensated by analyzing the degree and level of trust. Hence, analyzing the level and degree of perceived risk in the interaction is very important when making an informed interaction-based decision, apart from considering just the level of trust.

Also from an evaluation of the literature, it can be concluded that there have been various approaches proposed by researchers in the literature to model the perceived risk in an interaction. Approaches have been proposed which analyze the perceived risk in the security aspects of the interaction, and then utilize it for taking an interacting-based decision. Various other approaches have been proposed which determine the level of perceived risk in the interaction based on the level of trust and then utilize them for decision making. But from an critical evaluation of those approaches, no effort has been made by the researchers to devise a methodology for ascertaining the level of perceived risk, by considering its subcategories and characteristics for decision making. By the 'characteristics' of perceived risk we mean taking into consideration the context specific nature and the dynamic nature of risk while ascertaining it. By 'context specific nature of risk' we mean modeling the risk values in forming an interaction with an agent accurately according to the specific context and further in the detail criteria in that particular context of the interaction. By the 'dynamic nature of risk' we mean modeling the changes in the risk values of an agent as a result of change in its behavior over the period of time in the interaction. By 'decision making' we mean utilizing the level of determined perceived risk according to its subcategories and characteristics, and then utilize it to make an informed interacting-based decision.

In order to determine the perceived risk in an interaction according to its characteristics, it is imperative that the interaction for which it is being determined is analyzed according to these factors. This implies that the initiating agent of the interaction should consider the context and criteria specific and the various time scenario characteristics of its interaction with another agent while ascertaining each of the subcategories of perceived risk, in order to ascertain the perceived risk in the interaction according to its characteristics. Hence, a 'complete methodology' for ascertaining the perceived risk in a business interaction for decision making should consist of first identifying the subcategories of perceived risk according to its object of analysis in the area of discussion, then assess each of those subcategories according to the context specific, criteria specific and temporal characteristics of its interaction, then combine each of the subcategories to ascertain the level of perceived risk in the interaction, and then develop a decision making methodology based on the level of perceived risk in the interaction. It is important to note here that perceived risk in the interaction while decision making can be utilized efficiently only when it is a combined result of all its subcategories, rather than when it is a result of its partial subcategory, or a result of its subcategories sans the context specific, criteria specific and dynamic nature of risk.

Hence, the main inadequacy of the approaches in the literature to proposing a complete methodology for ascertaining the level of perceived risk in the interaction for decision making can be summarized as:

- Perceived risk has not been defined for it to be considered and analyzed in the domain of business industrial ecosystems interactions according to its subcategories and characteristics.

- There is no methodology proposed which determines each subcategory of perceived risk according to its characteristics, in the various time scenarios of the interaction. By the various 
time scenarios of the interaction, we mean the different time period to which the interaction might extend. It may be the case that the interaction might be limited to the current time or might extend till a point of time in the future.

- There is no methodology proposed for ascertaining the different levels and magnitudes of perceived risk present in the interaction, by combining its different subcategories determined according to the characteristics of the interaction.

- There is no methodology proposed for making an informed interaction-based decision, by considering the level of determined perceived risk in the interaction.

These issues need to be addressed and the concept of perceived risk needs to be considered apart from considering the level of trust and security by the initiating agent of the interaction in order to make an informed interaction-based decision in industrial ecosystems.

\section{SUMMARY}

Hence to summarize the broad issue in the current literature with regards to making an informed interaction-based decision in industrial ecosystems is that the interaction initiating agent needs to have a complete methodology by which it can make an informed interaction-based decision with another agent/s. By complete methodology we mean a methodology which assesses, quantifies and considers the level of risk along with considering the level of trust and security while making an informed interaction-based decision. Assessing and considering the level of risk in an interaction for decision making is important as it helps to analyze the level and degree of loss along with the associated consequences to the initiating agent in forming an interaction with another agent. In this paper, we have identified and listed the issues that need to be addressed in the current literature for making an informed interaction-based decision.

\section{REFERENCES}

[1] Elizabeth Chang, Tharam Dillon, and F. K. Hussain, Trust and Reputation for Service-Oriented Environments. West Sussex, England: John Wiley \& Sons, Ltd, 2006.

[2] Seth Schoen, "Trusted Computing: Promise and Risk," 2003.

[3] Eleni Koutrouli and A. Tsalgatidou, "Reputation-Based Trust Systems for P2P Applications: Design Issues and Comparison Framework," in 3rd International Conference on Trust, Privacy \& Security in Digital Business (TrustBus'06), Krakow, Poland, 2006, pp. 152-161.

[4] H. R. Rao, "Study Shows Perceived Risk of Online Credit Purchases Linked to Trust, Familiarity with Intermediaries," in University of Buffalo, News Centre, 2007.

[5] Bo-chiuan Su, "Risk behavior of Internet shopping: Comparison of College Students' versus Non-Student Adults'," in Proceedings of the 5th international conference on Electronic Commerce (ICEC 2003), Pittsburgh, Pennsylvania 2003, pp. $181-185$

[6] Stefano Grazioli and S. L. Jarvenpaa, "Perils of Internet Fraud: An Empirical Investigation of Deception and Trust with Experienced Internet Consumers," IEEE Transactions on Systems, Man and Cybernetics, vol. 30, pp. 395-410, July 2000.
[7] Aaron M. Hoffman, "A Conceptualization of Trust in International Relations," European Journal of International Relations, vol. 8, pp. 375-401, 2002.

[8] R.C. Mayer, J.H. Davis, and D. F. Schoorman, "An Integrative Model of Organizational Trust," The Academy of Management Review, vol. 20, pp. 709-734, 1995.

[9] David Gefen, V. Srinivasan Rao, and N. Tractinsky, "The Conceptualization of Trust, Risk and Their Relationship in Electronic Commerce: The Need for Clarifications," in Proceedings of the 36th Hawaii International Conference on System Sciences (HICCS'03), Big Island, Hawaii, USA, 2003, pp. 192-201.

[10]Anwitaman Datta, Manfred Hauswirth, and K. Aberer, "Beyond "web of trust": Enabling P2P E-commerce," in IEEE International Conference on ECommerce (CEC'03), Newport Beach, CA, USA, 2003, pp. 303-312.

[11]Karl Aberer, Philippe Cudré-Mauroux, Anwitaman Datta, Zoran Despotovic, Manfred Hauswirth, Magdalena Punceva, and R. Schmidt, "PGrid: A Self-Organizing structured P2P system," ACM SIGMOD Record, vol. 32, pp. 29 - 33 September 2003.

[12]Karl Aberer and Z. Despotovic, "Managing trust in a Peer-2-Peer Information System," in Proceedings of the Tenth International Conference on Information and Knowledge Management (CIKM'01), Atlanta, Georgia, USA, 2001, pp. 310 - 317.

[13]Ernesto Damiani, Sabrina De Capitani di Vimercati, Stefano Paraboschi, and Pierangela Samarati, "Managing and Sharing Servents' Reputations in P2P Systems," IEEE Transactions on Knowledge and Data Engineering, vol. 15, pp. 840-854, July/August 2003.

[14]Ernesto Damiani, Sabrina De Capitani di Vimercati, Stefano Paraboschi, Pierangela Samarati, and F. Violante, "A reputation-based approach for choosing reliable resources in peer-to-peer networks," in Proceedings of the 9th ACM conference on Computer and communications security Washington, DC, USA 2002, pp. 207 - 216

[15]Farookh Khadeer Hussain, Elizabeth Chang, and T. S. Dillon, "Trustworthiness and CCCI metrics in P2P communication," Journal of Computer Systems: Science \& Engineering, vol. 19, pp. 173-190, 2004.

[16]Elizabeth Chang, Farookh Khadeer Hussain, and T. S. Dillon, "Trustworthiness Measure for e-Service," in Third Annual Conference on Privacy, Security and Trust (PST'05), New Brunswick, Canada, 2005.

[17]Xiaoqing Zheng, Zhaohui Wu, Huajun Chen, and Y. Mao, "A Scalable Probabilistic Approach to Trust Evaluation," in Fourth International Conference on Trust Management (iTrust'06), Pisa, Italy, 2006, pp. 423-438.

[18]Colin English and S. Terzis, "Gathering Experience in Trust-Based Interactions," in The Fourth International Conference on Trust Management (iTrust'06), Pisa, Italy, 2006, pp. 62-76.

[19]Fahim Akhter, Dave Hobbs, and Z. Maamar, "Determining the Factors which Engender Customer Trust in Business-to-Consumer (B2C) Electronic Commerce," in IEEE International Conference on E-Commerce Technology (CEC 2004), San Diego, CA, 2003.

[20]Audun Jøsang, Claudia Keser, and T. Dimitrakos, "Can We Manage Trust?," in Third International Conference on Trust Management (iTrust 2005), Rocquencourt, France, 2005, pp. 93-107.

[21]Lewis Hassell, "Affect and Trust," in Third International Conference on Trust Management (iTrust'05), France, 2005, pp. 131-145.

[22]Siani Pearson, Marco Casassa Mont, and S. Crane, "Persistent and Dynamic Trust: Analysis and the Related Impact of Trusted Platforms," in Third International Conference on Trust Management (iTrust'05), France, 2005, pp. 355-363. 